\title{
An inverse-Warburg effect and the origin of Alzheimer's disease
}

\author{
Lloyd A. Demetrius • David K. Simon
}

Received: 2 May 2012/ Accepted: 28 September 2012/Published online: 20 October 2012

(C) Springer Science+Business Media Dordrecht 2012

\begin{abstract}
Glycolysis and oxidative phosphorylation (OxPhos) are the two major mechanisms involved in brain energetics. In this article we propose that the sporadic forms of Alzheimer's disease (AD) are driven by age-related damage to macromolecules and organelles which results in the following series of dynamic processes. (1) Metabolic alteration: Upregulation of OxPhos activity by dysfunctional neurons. (2) Natural selection: Competition for the limited energy substrates between neurons with normal OxPhos activity [Type (1)] and dysfunctional neurons with increased OxPhos [Type (2)]. (3) Propagation, due to the fact that Type (1) neurons are outcompeted for limited substrate by Type (2) neurons which, because of increased ROS production, eventually become dysfunctional and die. Otto Warburg, in his studies of the origin of cancer, discovered that most cancer cells are characterized by an increase in glycolytic activity-a
\end{abstract}

L. A. Demetrius $(\bowtie)$

Department of Organismic and Evolutionary Biology,

Harvard University, Cambridge, MA 02138, USA

e-mail: 1demetr@oeb.harvard.edu

L. A. Demetrius

Max Planck Institute for Molecular Genetics, Ihnestraße, 14195 Berlin, Germany

D. K. Simon

Department of Neurology, Beth Israel Deaconess Medical

Center and Harvard Medical School, Boston

MA 02215, USA

e-mail: dsimon1@bdmic.harvard.edu property which confers a selective advantage in oncologic environments. Accordingly, we propose the term "inverse-Warburg effect" to describe increased OxPhos activity-a property which we propose confers a selective advantage in neuronal environments, and which we hypothesize to underlie the shift from normal to pathological aging and subsequent $\mathrm{AD}$.

Keywords Oxidative phosphorylation - Glycolysis · Metabolism · Natural selection · Aging

\section{Introduction}

Alzheimer's disease (AD) is an age-related disorder characterized by progressive memory loss, impairment in cognitive skills and ultimately death. The principal pathologic signatures of the disease are the presence of extracellular deposits of amyloid-B as senile plaques and intracellular neurofibrillary tangles particularly in the neocortex, hippocampus and other subcortical regions which are critical for cognitive functions (Lin and Beal 2006).

Although inheritance of certain susceptibility genes increases the risk of the disease and rare monogenic forms exist, the large majority of $\mathrm{AD}$ cases occur sporadically with an incidence that rises dramatically with age (Lin et al. 2002). 
This article proposes a new model for the origin of the sporadic form of $\mathrm{AD}$ based on age-related metabolic dysregulation, and the bioenergetic strategies adopted by neurons in an attempt to ensure that the brain's supply of energy remains within certain limits.

The model is based on an analysis of the two main modes of energy production-glycolysis and oxidative phosphorylation. Energy metabolism in the brain is quite distinct from energy metabolism in other organs. Brain energy metabolism involves both astroytes and neurons (Pellerin and Magistretti 1994; Pellerin and Magistretti 2004a, b). Both cells utilize glucose as an energy source. In astrocytes, the glucose is metabolized anaerobically to lactate which is released into the extracellular milieu. In neurons, by contrast, the predominant mode of energy production is by OxPhos. Neurons are unable to increase glycolysis due to the lack of activity of glycolysis promoting enzymes (Bolaños et al. 2010), hence when brain cells are stressed, the associated increased demand for energy requires the upregulation of glycolyis in astrocytes. This results in an increased production of lactate which, upon release into the extracellular milieu, provides an energy substrate for neurons with upregulated OxPhos activity (Pellerin and Magistretti 2004a, b).

In our model we postulate that metabolic dysregulation is the primary cause of AD. This dysregulation, we contend, is a natural result of the aging process and derives from the cumulative effects of random perturbations of the cellular components involved in energy metabolism. The resulting damage will lead to a decreased efficiency of energy metabolism, and thus to a reduction in ATP production. We propose that $\mathrm{AD}$ is a consequence of compensatory alterations in brain energy metabolism that occur in response to diminished energy production in damaged neurons. The following three dynamic processes are proposed.

(1) Metabolic alteration: An upregulation of OxPhos activity in impaired neurons to compensate for the diminished efficiency of energy production.

(2) Natural selection: Competition for lactate (generated by astrocytes) between neurons with normal OxPhos activity, and impaired neurons with upregulated OxPhos activity.
(3) Propagation: Neurons with initially normal OxPhos activity are outcompeted for the limited substrate by neurons with upregulated OxPhos.

The model contends that upregulation of OxPhos in neurons will tend to propagate to other neurons due to the selective advantage that this provides in the neuronal microenvironment in which energy substrate is limited. We will call this upregulation of OxPhos activity in neurons-a bioenergetics response to diminished efficiency of energy production-the inverse-Warburg effect.

The use of the term "inverse-Warburg" can be rationalized by the fact that an analogous process - the upregulation of glycolysis-is now considered as a primary cause of certain sporadic forms of cancer. Warburg in 1924 proposed that the disease is a consequence of impairment in the OxPhos mechanism of cells and the upregulation of glycolysis, now called the Warburg effect, to compensate for the diminished energy (Warburg 1931).

The various hallmarks of cancer, Warburg argued, are a result of the glycolytic phenotype. An evolutionary rationale of the Warburg effect was given in Demetrius et al. (2010), where we invoked a selection principle (Demetrius 1997) to show that in oncologic microenvironments, cells with upregulated glycolysis will outcompete cells that depend on OxPhos activity, due to the higher efficiency of glycolytic cells in appropriating resources when there are large variations in abundance of those resources.

The model distinguishes between normal aging, which is characterized by a quasi-stable state with mild cognitive impairment, and pathological aging, characterized by an uncontrolled cycle of impairment, neuronal loss and concomitant dementia (Wang and Michaelis 2010).

Normal aging is driven by molecular instability leading to metabolic dysregulation, metabolic compensation, and ultimately competition for substrates between neurons with different levels of OxPhos activity. The outcome of this competition will lead to a neuronal population defined by a distribution of healthy and degenerate neurons. Cellular maintenance systems, such as DNA repair and anti-oxidant enzymes, will ensure the constancy of this neuronal distribution which defines a functional but mildly impaired network. 
The inverse-Warburg hypothesis proposes that pathological aging is induced by the disruption of the quasi-stable state due to the overextension of the cellular processes which exist to mitigate the effect of fluctuations in the concentration of reactive oxygen species (ROS). The collapse of ROS homeostasis induced by this disruption will result in an uncontrolled cycle of metabolic impairment, with propagation of neuronal dysfunction to other neighboring neurons, ultimately resulting in the spread of pathology and immense neuronal loss.

Our model is an application of the metabolic stability theory of aging (Demetrius 2004, 2006; Brink et al. 2009). The term metabolic stability describes the rate at which critical cellular metabolites return to their steady state values after a random perturbation in the chemical reaction rates. A rapid return rate implies strong homeostatic regulation and is associated with a high efficiency with which cells transform the energy source of nutrients into chemical energy.

Aging, according to the metabolic stability theory, results from a gradual decline in metabolic stability once the state of reproductive maturity is attained (Demetrius 2004; Brink et al. 2009). The primary cause of this decline in homeostasis is the increase in thermodynamic entropy, a measure of the extent to which energy is dispersed or distributed among the molecular components of the cell. This increase is inherent in the intrinsic thermodynamic instability of the bioorganic molecules, DNA, RNA and proteins that are involved in cellular metabolism. The functions of these organic molecules are contingent on their thermodynamic stability, which can become compromised as a result of conformational alterations, and covalent modifications of the molecules (Hayflick 2007).

As the organism ages, the impaired efficiency of energy metabolism will result in a decrease in evolutionary entropy, a measure of the rate at which energy is appropriated from the external environment and distributed between the cellular components. This will result in a decrease in metabolic stability and a deviation in energy levels from the homeostatic condition - a state which is critical for maintaining the integrity and viability of the cell (Demetrius 2004).

The metabolic stability theory of aging recognizes both the potentially deleterious effect of ROS and their critical role as a regulator of physiological functions (Finkel 1998). Accordingly, the theory contends that it is the homeostatic condition, namely, the ability to maintain ROS and other critical metabolites at their steady state levels which is the critical determinant of the rate of aging.

The application of the metabolic stability theory of aging to our model of the origin of $\mathrm{AD}$ is based on the previously stated premises of metabolic alteration, natural selection, and propagation, which ultimately lead from a transition from normal to pathological aging in which neuronal loss and dementia result from an uncontrolled cycle of metabolic impairment due to the selective advantage of neurons with upregulated OxPhos in terms of competing for limited energy substrates.

\section{Bioenergetics}

Modes of energy transduction

Energy is invoked in all active cellular processes. ATP is the main energy currency in living organisms and must be continually available to maintain cell viability.

The standard energy of ATP under physiological conditions is precisely maintained in all cells between -53 and $-60 \mathrm{~kJ} / \mathrm{mol}$ (Seyfried and Shelton 2010; Veech et al. 1979). The energy is derived from two types of processes; OxPhos which provides about $88 \%$ of the total energy, and substrate level phosphorylation (mainly glycolysis) which contributes the remaining $12 \%$.

Accordingly, in order to maintain viability of cells, an increase in substrate level phosphorylation may occur in cells subject to mitochondrial injury, whereas a compensatory increase in OxPhos activity may occur in cells whose glycolytic enzymes are inactive.

Oxidative phosphorylation occurs within the mitochondria. Electrons from oxidative substrates are transferred to oxygen via a series of redox reactions to generate water. In this process, protons are pumped from the matrix across the mitochondrial inner membrane through a set of respiratory complexes. When protons return to the mitochondrial matrix down their electrochemical gradient, ATP is synthesized via the enzyme ATP synthase. Energy production in this context is electrical. The rate of energy production, the metabolic rate, is determined by the conductance of the biomembrane and the electromotive potential across the membrane (Nicholls and Ferguson 2002). 
Glycolysis occurs within the cytosol. The glycolytic enzymes occur in relatively stable multienzyme complexes with metabolites passed on from one active site to the next without exchanging with the bulk cytoplasm. Energy production in this case is chemical. The rate of energy production is now determined by the activity of the glycolytic enzymes in the cytosol.

The effect of the mechanism of energy transduction-OxPhos or glycolysis on the metabolic rate, was investigated in (Demetrius et al. 2010). There it was shown that the metabolic rate $P$ is allometrically related to cell size, $W$, according to the equation

$P=\alpha W^{d / d+1}$

where the integer $d$ in the scaling exponent satisfies the relation $1<d<\infty$. The proportionality constant $\alpha$ is determined by the mode of energy transduction.

In the case of OxPhos, the proportionality constant $\alpha$ is determined by the proton gradient across the mitochondrial membrane, a quantity which is largely determined by the phospholipid composition of the membrane (Nicholls and Ferguson 2002). This dependency entails that the metabolic rate of cells utilizing OxPhos can be modified by chemicals which alter the phospholipid composition.

The parameter $\alpha$ in systems driven by substrate phosphorylation is determined by the mean reaction rate of the glycolytic enzymes. This dependency means that the metabolic rate of cells utilizing glycolysis can be modulated by regulating the activity of enzymes which control glycolysis.

\section{Brain energy metabolism}

The energy requirement of the brain is determined largely by neuronal demand for energy to maintain ion gradients across the plasma membranes that are critical in neurotransmission (Bolaños et al. 2010).

Brain energy metabolism involves both astrocytes and neurons (Pellerin and Magistretti 1994). Both neurons and astrocytes utilize glucose as an energy source. In astrocytes, the predominant mode of energy production is by glycolysis. Glucose is metabolized anaerobically to lactate, and the lactate is released into the extracellular milieu. In neurons by contrast the predominant mode of energy production is by OxPhos. This condition entails that when brain cells are stressed, the associated increased demand for energy requires the upregulation of glycolysis in the astrocytes, resulting in an increased production of lactate, thus providing substrate to drive a concomitant upregulation of OxPhos activity in the neurons using lactate as the energy substrate (Pellerin and Magistretti 1994; Bolaños et al. 2010).

\section{Natural selection and the origin of AD}

Empirical studies show that an important factor in early pathology is mitochondrial dysfunction and oxidative stress (Lin and Beal 2006; Mattson and Magnus 2006).

Oxidative stress, which refers to damage caused by high levels of reactive oxygen species, is known to precede the onset of symptoms such as episodic memory loss (Mattson and Magnus 2006). This feature also occurs before the formation of neurofibrillary tangles and senile plaques. This fact supports (but does not prove) the notion that oxidative damage could be a cause of the pathological hallmarks of disease.

Based on the metabolic stability theory of aging (Demetrius 2004), we propose that, as the organism ages, the accumulation of random perturbations in the metabolic network eventually results in impaired mitochondrial function. The impairment in the energy transducing organelles will result in reduced efficiency of the neurons to transform the energy source of neuronal substrates into chemical energy (ATP). This decrease in metabolic efficiency entails a decrease in the evolutionary entropy of the neuron, that is the diversity of the pathways of energy flow within the cell.

The model contends that there exist two modes of metabolic alteration, leading to Type (1) and Type (2) cells, which may compensate for the decline in ATP production due to the aging process. We characterize these alterations as follows:

(a) Type (1): This class includes neurons with normal or diminished OxPhos activity. These neurons, in view of the diminished energy production limit their signaling capacity while conserving the housekeeping functions which are needed for survival.

(b) Type (2): This class includes neurons with upregulated OxPhos capacity. These neurons 
produce sufficient energy to maintain signaling capacity. However, the upregulation of OxPhos entails an enhanced ROS production with possible deleterious effects.

Our model postulates that the outcome of these agerelated changes induced by metabolic dysregulation and metabolic alteration will now depend on natural selection, that is: the competition between Type (1) and Type (2) neurons for the lactate released by the astrocytes to be used as an energy substrate by neurons.

Our analysis of the outcome of competition for substrates between the two cell types derives from an evolutionary principle which predicts that in a neuronal microenvironment characterized by an energy source which is constant and limited in abundance, neurons with increased OxPhos activity will have a selective advantage over neurons with normal or diminished OxPhos activity.

The steady state condition of this selection process is a quasi-stable population consisting of neurons with two levels of metabolic activity: relatively healthy neurons with normal OxPhos, and mildly dysfunctional neurons with a compensatory increase in OxPhos activity. The dysfunction of these neurons, in the model, is a consequence of the increased ROS production which may arise from the upregulation of OxPhos.

This quasi-stable condition, an inevitable consequence of normal aging, will be accompanied by various levels of cognitive decline such as defects in short term memory. The model contends that the transition to neuronal loss, and the accelerated degeneration that characterizes pathological aging with more severe defects in memory and cognition, is contingent on the disruption of the quasi-stable condition. This collapse, we propose, will be due to the breakdown in the homeostasic mechanisms to regulate the concentration of metabolites which will maintain the Type (2) neurons in a dynamic steady state. A dysregulation in homeostatic function entails a shift from a gradual linear decline to an accelerated degeneration due to the increased intensity of selection for neurons with high OxPhos activity. The analytical support for this qualitative argument revolves around recent investigations on the dynamics of competition between populations, and a general evolutionary tenet, called the entropic selection principle (Demetrius 1997).

\section{The dynamics of selection: general considerations}

Competition between genotypically related populations is the principal process which governs evolutionary change. The analytical characterization of the laws which determine the outcome of competition is critical for understanding the origin and evolution of diversity at all levels of biological organization: molecular, cellular, and organismic.

The Malthusian principle and the entropic selection principle

The efforts to derive a principle of selection goes back to R. A. Fisher (1930), who proposed the population growth rate, now called the Malthusian parameter, as the demographic variable which predicts the outcome of natural selection. According to Fisher, the dynamics of competition is characterized by the Malthusian selection principle. This is described by the following rule:

The outcome of competition is a deterministic process predicted by the population growth rate.

Although intuitively one might expect this statement to be true, it is not always consistent with empirical observations. For example normal cells can grow much faster than tumor cells during normal repair. However, tumor cells are known to outcompete normal cells in the oncologic microenvironment. This observation, and experimental findings in related competitive contexts, indicate that the outcome of selection depends on a more complex suite of conditions.

The problem regarding competition between variant and resident populations was finally resolved when it was shown that the outcome of selection is not, as previously thought, determined by the population growth rate, but instead by population stability; that is the rate at which the population returns to the steady state condition after a random perturbation in the individual demographic variables (Demetrius 1997; Demetrius and Gundlach 2000).

Demographic stability can be described in terms of a statistical parameter called evolutionary entropy. This quantity, a dynamic analogue of the GibbsBoltzmann entropy in statistical thermodynamics, is a generic property that describes the rate at which 
energy is appropriated from the external environment and distributed between the various microstates of the macroscopic system.

Evolutionary entropy, in the case of a demographic system defined by a population of replicating cells, is a measure of the degree of synchrony of the population. A system in which each cell in the population passes through the different stages of the cell cycle at the same rate has zero entropy. A system in which the cells traverse the different stages of the cell cycle at different rates has positive entropy. The analytic expression for evolutionary entropy in cellular populations is

$S=-\sum_{j=1}^{n} p_{j} \log p_{j}$

Here $n$ denote the number of stages of the cell cycle, and $p_{j}$ is the probability that the mother of a randomly chosen daughter cell is in stage $j$ of the cycle.

The relation between evolutionary entropy and demographic stability is the basis for representing the dynamics of selection using entropy as the parameter which determines competitive outcome. This property can be qualitatively annotated by the following rule:

The outcome of selection between an incumbent and a variant type is a stochastic process which is predicted by evolutionary entropy and conditioned by the resource abundance and resource diversity.

The rule, called the entropic selection principle, delineates the relative effect of resource abundance and resource diversity on the outcome of selection. Resource abundance exerts a stronger effect than resource diversity on the selective dynamics. Indeed, the influence of resource diversity on the outcome of competition decreases as the population size increases (Demetrius et al. 2010).

In particular, when the population size is large, effectively infinite, the outcome of selection will be regulated uniquely by resource abundance. In this case the entropic selection principle can be qualitatively described by the following rules.

(a) When the resource abundance remains constant and is limited, types with larger entropy will have a selective advantage and increase in frequency. (b) When resources undergo large variations in abundance, types with smaller entropy will have a selective advantage and increase in frequency.

The dynamics of competition: neuronal populations

The entropic selection principle can be adduced to analyze the outcome of competition between neuronal populations with different levels of OxPhos activity.

A cell can be considered as a metabolic network, a population of interacting substrates, enzymes, and products. Evolutionary entropy in the context of this model refers to the diversity of metabolic pathways that describe the transformation of substrates into products.

A network defined by a unique cycle of reactions transforming substrate to products has zero entropy. The entropy increases as the number of possible reaction cycles in the network increases. The application of the entropic selection principle to the analysis of competition between neuronal populations rests on the analytical fact that evolutionary entropy is analytically related to the metabolic rate (Demetrius et al. 2009). We have,

$S=a+\log P$

where $a$ is a numerical constant, and $P$ denote the metabolic rate.

We can appeal to Eq. (3) to express the entropic selection principle in terms of an equivalent tenet: the metabolic selection principle (Demetrius et al. 2010).

The outcome of selection is a stochastic process predicted by the metabolic rate and contingent on the resource abundance and resource diversity.

When the population size is sufficiently large so that stochastic effects which derive from population size can be ignored, the selection principle can be qualitatively described as follows:

(a) When resources are constant and limited, neurons with higher metabolic rate will have a selective advantage and hence a higher survivorship.

(b) When resources are subject to large variation in abundance, neurons with lower metabolic rate 
Table 1 Comparison: normal aging-pathological aging

\begin{tabular}{|c|c|c|}
\hline Characteristics & Normal aging & Pathological aging \\
\hline Clinical & $\begin{array}{l}\text { Mild defects in short term memory, working } \\
\text { memory and processing speed; mildly impaired } \\
\text { spatial memory }\end{array}$ & $\begin{array}{l}\text { More severe defects in short term memory, defects in long } \\
\text { term memory, personality changes, more severe loss of } \\
\text { spatial memory }\end{array}$ \\
\hline Structural & Medial temporal lobe volume relatively preserved & Prominent volume loss (atrophy) of medial temporal lobes \\
\hline Molecular & $\begin{array}{l}\text { Mild accumulation of amyloid plaques and } \\
\text { neurofibrillary tangles }\end{array}$ & $\begin{array}{l}\text { More severe and diffuse accumulation of amyloid plaques and } \\
\text { neurofibrillary tangles }\end{array}$ \\
\hline Cellular & Neuronal dysfunction; impaired synaptic function & Neuronal loss, loss of dendritic branching, synaptic loss \\
\hline
\end{tabular}

will now have a selective advantage and hence a higher survivorship.

The metabolic selection principle pertains immediately to the analysis of competition between neurons with increased OxPhos activity, and neurons with normal or diminished OxPhos activity. Neurons with increased OxPhos will necessarily be described by an increased metabolic rate. Consequently, when the available resources are constant and limited, this class of neurons will appropriate the resources more efficiently. Types with high OxPhos will have an enhanced viability when compared with types with low OxPhos activity.

\section{Normal and pathological aging: metabolic alteration}

Physiological decline and increasing probability of death are characteristic features of aging. AD is agerelated. However, the disease is a property of pathological rather than normal aging in the brain. A comparison of the various properties which define these two modes of aging are described in Table 1.

The origin of $\mathrm{AD}$ is based on distinguishing between the molecular and metabolic alterations in neuronal dynamics which define the transition from normal to pathological aging.

\section{Normal aging}

Normal aging is driven by the loss of molecular fidelity, the generic term we use to describe abnormalities in mitochondrial DNA, the electron transport chain, and molecular defects in the phospholipid composition of the mitochondrial membrane. Molecular instability does not refer uniquely to defects in the mitochondria, but also to perturbation in the substrates and enzymes in the cytosol. This random and quasi-random perturbation in the machinery of energy production will result in diminished ATP production and compensatory metabolic alteration.

This process will result in the generation of two classes of neurons: Type (1), characterized by normal OxPhos activity, and Type (2) by a compensatory increase in OxPhos activity.

Figure 1 describes the two classes of neurons which compete for the substrate, namely lactate, which is produced by the astrocytes.

Since metabolic rate increases with an increase in OxPhos activity in the cells, we infer that the Type (2) neurons will have a higher metabolic rate than the Type (1) cells.

The selection principle can be applied to predict the outcome of competition between the two classes of neurons. The principle implies that, since the substrate used by the neurons is constant and limited, the Type (2) neurons will, in view of its higher metabolic rate, outcompete the Type (1) neurons for obtaining and using substrate.

The selective process will ultimately lead to a quasi-stable state consisting of neurons with diminishing levels of ATP production. Neurons with the highest frequency will be described by a high OxPhos activity.

This quasi-stable state will be maintained by two classes of processes:

(1) The action of anti-oxidant enzymes. The function of these enzymes is the control of the deleterious effects caused by an excessive production of ROS generated by neurons with high OxPhos activity 
Fig. 1 Astrocyte-neuronlactate-interaction

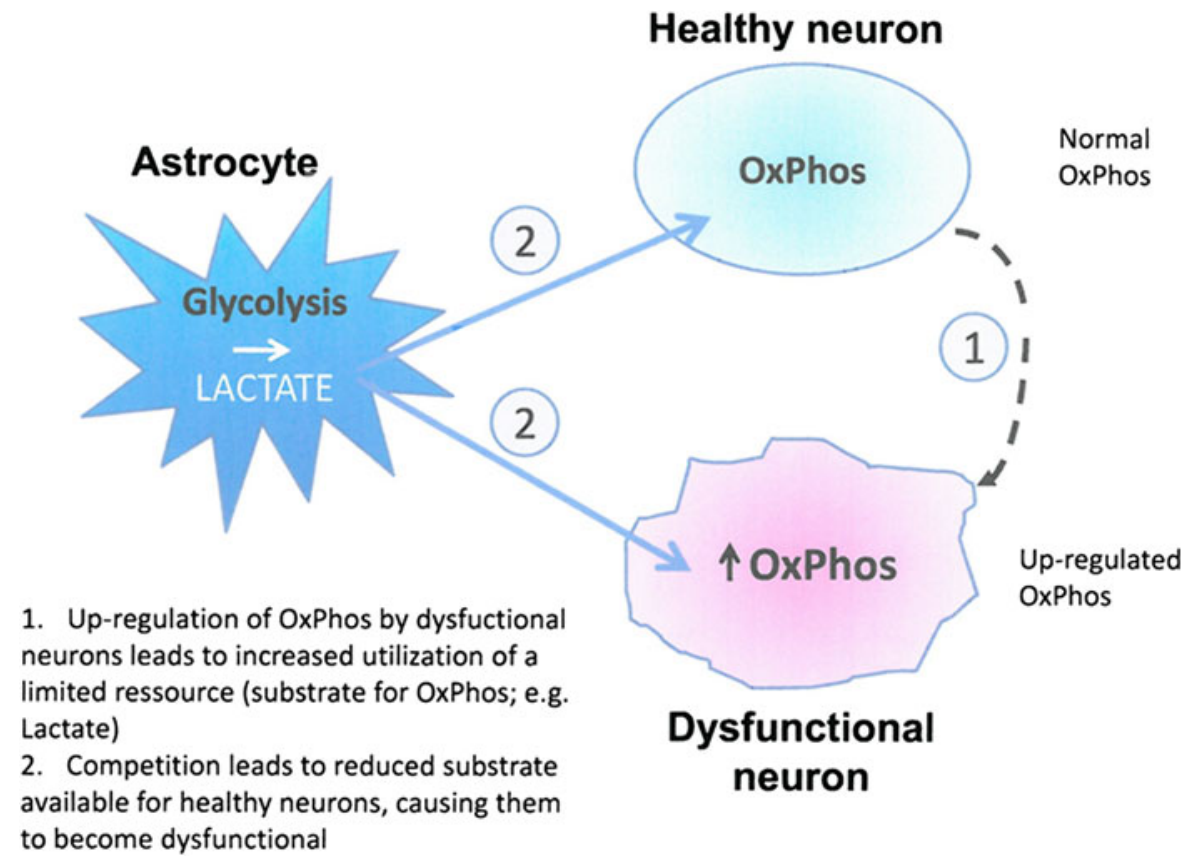

(2) The action of signal transduction enzymes. The function of these enzymes is to rectify the impairment in cell-cell signaling in neurons-a condition induced by the low production of ROS in neurons with low OxPhos activity.

Pathological aging

Pathological aging has its origin in instability at the level of brain regions and sectors. This level of instability derives from the propagation of dysfunctional neurons, due to their enhanced selective advantage, within the microenvironment of various tissues of the brain. The transition from normal aging to pathological aging is mediated by an increased intensity of selection due to the failure of anti-oxidant enzymes and signal transduction enzymes to maintain the quasistable state induced by normal aging.

The model predicts that factors associated with increased cellular stress will destabilize the steady state condition attained by the process of normal aging. The neuronal dysfunction which was initiated by molecular instability - an intrinsic property of normal aging-will no longer remain localized, but will now propagate to nearby neurons.

The nearby neurons, initially healthy, are outcompeted for substrate, and so will become unable to maintain stable ATP levels. These neurons thus develop metabolic impairment, eventually leading to compensatory up regulation of OxPhos and perpetuating a degenerative cycle. In this model, the initial trigger of the pathology is related to age, but the propagation is a result of an inverse-Warburg effect, and competition between healthy neurons with normal OxPhos activity-and diminished ATP production, and neurons with upregulated OxPhos activity-and enhanced ATP production.

There is evidence that OxPhos activity is downregulated in certain $\mathrm{AD}$ patients (Chandrasekaran et al. 1996). This physiological downregulation of OxPhos gene expression is observed primarily in mildly to moderately demented Alzheimer's patients. Moreover, the pattern of downregulation is mediated by changes in transcriptional and post-transcriptional regulatory factors. This reflects the reduced brain energy demand of patients with the disease. This downregulation is therefore not inconsistent with the upregulation of OxPhos which we have proposed as the metabolic alteration to compensate for the diminished ATP due to damaged neurons.

\section{Warburg and inverse Warburg}

Glycolysis, a relatively inefficient mechanism, and OxPhos, a highly efficient process, are the principal 
mechanisms which differentiated cells utilize to generate the energy needed for cellular processes.

Warburg effect and cancer

The Warburg effect is the observation that most cancer cells rely on aerobic glycolysis for energy production. Warburg postulated that the alteration from OxPhosthe predominant process in normal differentiated cells-to aerobic glycolysis is the primary cause of cancer and the determinant of the various hallmarks: self-sufficiency in growth signals, insensitivity to antigrowth signals, and limitless replicative potential that characterize the cancer phenotype.

The metabolic selection principle (Demetrius et al. 2010) was adduced to explain the selective advantage of cancer cells-using glycolysis, in competition with normal differentiated cells—using OxPhos. This selection principle thus resolved one of the paradoxes in oncology, namely the competitive success of cancer cells in spite of a less efficient metabolic mechanism. These observations have contributed to the understanding of cancer as a metabolic disease, and furnished support for Warburg's hypothesis.

\section{An inverse-Warburg effect and AD}

An inverse-Warburg effect, as promulgated in this article, pertains to the observation that neurons rely on OxPhos for energy production. The rate-limiting enzyme PFK1 for glycolysis is relatively inactive in neuronal cells in adults, and consequently ATP production in both healthy and degenerate neurons in adults is generated primarily by OxPhos.

In this article, we have postulated that the compensatory upregulation of OxPhos in neurons with impaired mitochondria is the primary cause of the transition from normal to pathological aging and concomitantly AD. We postulate that this metabolic shift is the critical determinant of the various hallmarks of $\mathrm{AD}$ : the severe accumulation of amyloid plaques, neuronal loss and loss of dendritic branching.

The metabolic selection principle, which we have cited previously to explain the proliferation of cancer cells, also plays a critical role in our analysis of the propagation of neuronal cells with the AD phenotype. The principle entails that cells with increased OxPhos activity will have a selective advantage in competition with relatively normal neuronal cells for substrate (lactate produced by the astrocytes).

Selective vulnerability of different brain regions and different neuronal populations within specific brain regions is dependent on many factors (Wang and Michaelis 2010). Some factors that influence selective vulnerability are disease specific, such as levels of expression of specific disease related proteins. In addition, it has been hypothesized that neuronal populations with high energy demands are preferentially vulnerable to degeneration in response to mitochondrial dysfunction. An inverse-Warburg theory is consistent with this hypothesis. Neurons vary considerably in terms of their energy demands, and thus in the degree to which mitochondrial OxPhos must be upregulated in response to even mild mitochondrial dysfunction in order to maintain adequate ATP levels. According to the inverse-Warburg hypothesis, upregulation of OxPhos is the first step that triggers the cascade of events leading to pathological aging. Consequently, there will be considerable regional variation in the vulnerability of neuronal populations to degeneration in response to mitochondrial dysfunction.

A systematic study of variation in neuronal vulnerability is reported in Mattson and Magnus (Mattson and Magnus 2006). These authors report that the prefrontal cortex is more vulnerable to neurodegeneration than the cerebellum. The prefrontal cortex has greater energy demands than the cerebellum, a fact which is consistent with our prediction. The entorhinal cortex has a more complex synaptic architecture than the hippocampus. Moreover, it degenerates much earlier in the course of the disease. This early degeneration is also consistent with our prediction regarding differential neuronal vulnerability.

Clearly differences in energy demands is not the only factor that determines regional variability to neurodegeneration, as different degenerative disorders associated with mitochondrial dysfunction (including AD, HD, PD, and ALS) exhibit distinct regional patterns of degeneration. These issues were reviewed recently by Wang and Michaelis (2010). Still, the inverse-Warburg hypothesis highlights that the relative energy demands, will be among the factors that affect selective vulnerability to neurodegeneration in response to mitochondrial dysfunction. 


\section{Empirical considerations}

The central result of our analytic model of the energetic demands of the aging brain is a new proposal for the origin of $\mathrm{AD}$, namely: The primary cause of $\mathrm{AD}$ is an enhanced upregulation of OxPhos activity in neurons. According to our model, this upregulation is due to competition between neurons for the lactate produced by glycolytic activity in astrocytes, and the selective advantage of neurons with enhanced OxPhos activity in the neuronal-astrocytic microenvironment. Under normal conditions, levels of lactate produced through glycolysis in astrocytes are very low and highly variable. With pathological aging, there is an upregulation of glycolysis such that lactate levels become more constant, although still are limiting as an energy substrate for neurons. When a critical energy substrate such as lactate is both limited and constant, then neurons with a high metabolic rate (i.e. Type (2) neurons) will have a competitive advantage over neurons with normal levels of OxPhos. Thus, the Inverse Warburg Hypothesis proposes that upregulation of glycolysis in astrocytes increases the selective advantage of Type (2) neurons that have upregulated OxPhos as a compensatory strategy. The OxPhos upregulation transpires to maintain cellular energy levels in the face of inefficient mitochondrial energy metabolism due to mitochondrial dysfunction. A byproduct of this upregulation of OxPhos is an increased production of ROS, which has potentially deleterious consequences.

Several models have already been proposed for the primary cause of AD (Hardy and Allsop 1991; Selkoe 2000). A molecule which has emerged as a critical factor in $\mathrm{AD}$ is beta amyloid. This has led to the proposition that beta amyloid production is the prime cause of AD. In rare genetic forms, a genetic mutation induces amyloid deposition. In sporadic AD, we don't yet fully understand the key factors that influence amyloid deposition. But the Inverse Warburg Hypothesis has relevance to that question.

Recent investigations on the relation between neuronal oxidative metabolism and astrocyte glycolysis, and the effect of astrocyte glycolysis on the activity of beta amyloid production furnishes an experimental framework for evaluating the prediction that the upregulation of OxPhos activity in neurons is the primary cause of beta amyloid deposition in sporadic AD. The Inverse Warburg Hypothesis proposes that increased ROS production by Type (2) neurons will promote the accumulation of beta amyloid, and thus contribute to the spread of the pathological process of $\mathrm{AD}$. The competitive advantage of Type (2) neurons will contribute to maintaining the limited state of lactate levels, ultimately leading to energy deficits for Type (1) neurons, which then will upregulate OxPhos and thus ultimately convert to Type (2) neurons, with their associated increased ROS production. This sequence of events will further promote the spread of beta amyloid deposition. Thus, our model contends that it is the upregulation of OxPhos activity which drives the production and/or deposition of beta amyloid in sporadic AD.

Certain aspects of the Inverse Warburg Hypothesis already are supported by experimental observations. Experimental studies using functional imaging techniques (Kasischke et al. 2004) have shown a tight coupling between oxidative metabolism in neurons and glycolysis in astrocytes. This is consistent with the astrocyte-neuron shuttle lactate hypothesis (Pellerin and Magistretti 2004a, b), and suggests that an upregulation of OxPhos activity in neurons can trigger the glycolytic activity in astrocytes: These cells will produce lactate which now constitutes a new energy source for neurons. In addition, studies reported in Allaman et al. (2010) indicate that increased glycolytic activity in astrocytes can result in the increased production of beta amyloid as well as inducing metabolic dysregulation.

This model makes additional predictions that can be experimentally tested. One prediction is that OxPhos activity will be increased in a subset of neurons in vulnerable brain regions in AD. Upregulation of glycoylsis within astrocytes varies across different brain regions, and this distribution mirrors the distribution of beta amyloid accumulation. The Inverse Warburg Hypothesis predicts that upregulation of OxPhos within neurons will similarly parallel the distribution of beta amyloid accumulation. This cannot be adequately tested by functional imaging studies or metabolic analysis of tissue homogenate, which are influenced by the metabolic state of many cell types (neurons, glia, and others), but rather requires analysis of the metabolic state specifically within neurons. Anti-oxidants already hypothesized to have potential protective value in $\mathrm{AD}$ (but not yet demonstrated unequivocally in clinical trials), but the Inverse Warburg Hypothesis also suggests that experimental 
manipulations that increase glycolysis in astrocytes also could limit or prevent AD pathology if it is upregulated to an extent that lactate no longer was limiting as an energy substrate for neurons. Other strategies that provide ample energy substrate for neurons such that the substrate is no longer limiting also would be predicted to limit the spread of $\mathrm{AD}$ pathology.

\section{Therapeutic implications: suppressing the inverse- Warburg effect}

The metabolic stability theory of aging contends that the increase in probability of death associated with the aging process is the result of the cumulative effects of the random perturbations on mitochondrial function.

These perturbations will ultimately induce metabolic dysregulation in some neurons and result in compensatory upregulation of OxPhos-an inverseWarburg effect-ultimately contributing to the pathogenesis of AD. Accordingly, $\mathrm{AD}$ is an age-related disease and the transition towards the various hallmarks of the disease is a consequence of the aging process.

The model predicts that the transition towards $\mathrm{AD}$ is determined by the intensity of natural selection. Accordingly, impeding this transition may be modulated by regulating this evolutionary force. There are two principal strategies for reducing selection intensity:

I. Regulating the concentration of the substrate lactate: neurons utilize lactate as the energy source under conditions of cellular stress. The lactate is produced by glycolysis in the astrocytes. An increase in the activity of of the glycolytic enzyme in astrocytes will increase the abundance of the energy source lactate, and thereby reduce the selective advantage of the Type (2) cells.

II. Modulating the damage due to the collapse of homeostasis in ROS: Both very high and very low concentration of ROS can have deleterious effects and thereby drive the system towards a state in which neuronal dysfunction is propagated to nearby neurons.

(a) The effect of high concentration of ROS: This condition can be diminished by the upregulation of antioxidant enzymes e.g. catalase or superoxide dismutase.

(b) The effect of low concentration of ROS: Cell signaling is compromised when concentration of ROS is significantly reduced. Thus, upregulation of antioxidant activities must be done cautiously to avoid disruption of oxidant signaling, with a focus on strategies to maintain stable levels of ROS rather than to achieve maximal reduction of ROS levels.

The theory we have developed in this article provides an evolutionary rationale for the possible effectiveness of these therapeutic strategies, and suggest furthermore how related enzymes may have similar therapeutic effects. Thus, our hypothesis of the role of the inverseWarburg effect in AD leads to testable predictions regarding novel therapeutic strategies for AD.

\section{Conclusion}

Alzheimer's disease is a neurodegenerative disorder, characterized by progressive cognitive decline and pathologically by the presence of senile plaques in the brain. This article explains how competition for a limited resource (astrocyte derived lactate as a substrate for neuronal OxPhos) can lead to deleterious effects on initially healthy neurons in the vicinity of other neurons that have mitochondrial dysfunction. The model predicts that the severity of the disease will be a consequence of the intensity of natural selection involving two classes of neurons: Type (1) neurons with normal levels of OxPhos activity and Type (2) neurons, cells with mitochondrial dysfunction and compensatory increases in levels of OxPhos.

Our analysis predicts that the intensity of selection can be reduced by metabolic interventions that (a) alter the neuronal microenvironment by increasing astrocytic lactate production such that lactate no longer is a limiting resource in terms of neuronal energy production via OxPhos; and (b) reduce the deleterious effects of large variations in ROS by the cautious use of antioxidants to modulate the effects of high ROS.

Acknowledgments We received constructive criticism from Paul Agutter, Luc Pellerin, and Tom Seyfried. We are very grateful for their help and advice. LAD receives support for his 
research from the Max Planck Society, DKS is supported by NIH (NINDS and NIA) and the Michael J. Fox Foundation.

Conflict of interest The authors declare that they have no competing interests to disclose.

\section{References}

Allaman I, Gavillet M, Laroche T, Viertl D, Lashuel HA, Magistretti PJ (2010) Amyloid-beta aggregates cause alterations of astrocytic metabolic phenotype: impact on neuronal viability. J Neurosci 30(9):3326-3338

Bolaños J, Almeida A, Moncada S (2010) Glycolysis: a bioenergetic or a survival pathway? Trends Biochem Sci 35(3): $145-149$

Brink TC, Demetrius LA, Lehrach H, Adjaye J (2009) Agerelated transcriptional changes in gene expression in different organs of mice support the metabolic stability theory of aging. Biogerontology 10(5):549-564

Chandrasekaran K, Hatanpää K, Brady DR, Rapoport SI (1996) Evidence for physiological down-regulation of brain oxidative phosphorylation in Alzheimer's disease. Exp Neurol 142(1):80-88

Demetrius LA (1997) Directionality principles in thermodynamics and evolution. Proc Nat Acad Sci USA 94(8): 3491-3498

Demetrius LA (2004) Caloric restriction, metabolic rate, and entropy. J Gerontol A Biol Sci Med Sci 59(9):B902-B915

Demetrius LA (2006) Aging in mouse and human systems: a comparative study. Ann N Y Acad Sci 1067:66-82

Demetrius LA, Gundlach VM (2000) Game theory and evolution: finite size and absolute fitness measures. Math Biosci 168(1):9-38

Demetrius LA, Legendre S, Harremöes P (2009) Evolutionary entropy: a predictor of body size, metabolic rate and maximal life span. Bull Math Biol 71(4):800-818

Demetrius LA, Coy JF, Tuszynski JA (2010) Cancer proliferation and therapy: the Warburg effect and quantum metabolism. Theor Biol Med Model 7:2

Finkel T (1998) Oxygen radicals and signaling. Curr Opin Cell Biol 10(2):248-253
Fisher RA (1930) The genetical theory of natural selection, vol 154. Clarendon Press, Oxford

Hardy J, Allsop D (1991) Amyloid deposition as the central event in the aetiology of Alzheimer's disease. Trends Pharmacol Sci 12(10):383-388

Hayflick L (2007) Biological aging is no longer an unsolved problem. Ann N Y Acad Sci 1100:1-13

Kasischke KA, Vishwasrao HD, Fisher PJ, Zipfel WR, Webb WW (2004) Neural activity triggers neuronal oxidative metabolism followed by astrocytic glycolysis. Science 305:99-103

Lin MT, Beal MF (2006) Mitochondrial dysfunction and oxidative stress in neurodegenerative diseases. Nature 443(7113): 787-795

Lin MT, Simon DK, Ahn CH, Kim LM, Beal MF (2002) High aggregate burden of somatic mtDNA point mutations in aging and Alzheimer's disease brain. Hum Mol Genet 11(2):133-145

Mattson MP, Magnus T (2006) Aging and neuronal vulnerability to oxidative stress in the brain. Front Aging Neurosci 2:1-13

Nicholls DG, Ferguson SJ (2002) Bioenergetics 3. Academic Press, London

Pellerin L, Magistretti PJ (1994) Glutamate uptake into astrocytes. Neurobiology 91(22):10625-10629

Pellerin L, Magistretti PJ (2004a) Neuroscience. Let there be (NADH) light. Science 305(5680):50-52

Pellerin L, Magistretti PJ (2004b) Neuroenergetics: calling upon astrocytes to satisfy hungry neurons. Neuroscientist 10(1): $53-62$

Selkoe DJ (2000) Toward a comprehensive theory for Alzheimer's disease. Hypothesis: Alzheimer's disease is caused by the cerebral accumulation and cytotoxicity of amyloid beta-protein. Ann N Y Acad Sci 924:17-25

Seyfried TN, Shelton LM (2010) Cancer as a metabolic disease. Nutr Metab 7:7

Veech RL, Lawson JW, Cornell NW, Krebs HA (1979) Cytosolic phosphorylation potential. J Biol Chem 254(14): 6538-6547

Wang X, Michaelis EK (2010) Selective neuronal vulnerability to oxidative stress in the brain. Front Aging Neurosci 2:12

Warburg O (1931) The metabolism of tumors. Academic Press, New York 